AcCepted to ApJ: September 10, 2013

Preprint typeset using $\mathrm{LAT}_{\mathrm{E}} \mathrm{X}$ style emulateapj v. 04/17/13

\title{
A SEARCH FOR PULSATIONS IN SHORT GAMMA-RAY BURSTS TO CONSTRAIN THEIR PROGENITORS
}

\author{
S. Dichiara ${ }^{1}$, C. Guidorzi ${ }^{1}$, F. Frontera ${ }^{1,2}$, L. Amati $^{2}$ \\ ACCePted to ApJ: September 10, 2013
}

\begin{abstract}
We searched for periodic and quasiperiodic signal in the prompt emission of a sample of 44 bright short gamma-ray bursts detected with Fermi/GBM, Swift/BAT, and CGRO/BATSE. The aim was to look for the observational signature of quasiperiodic jet precession which is expected from black hole-neutron star mergers, but not from double neutron star systems. Thus, this kind of search holds the key to identify the progenitor systems of short GRBs and, in the wait for gravitational wave detection, represents the only direct way to constrain the progenitors. We tailored our search to the nature of the expected signal by properly stretching the observed light curves by an increasing factor with time, after calibrating the technique on synthetic curves. In none of the GRBs of our sample we found evidence for periodic or quasiperiodic signals. In particular, for the 7 unambiguously short GRBs with best $\mathrm{S} / \mathrm{N}$ we obtained significant upper limits to the amplitude of the possible oscillations. This result suggests that BH-NS systems do not dominate the population of short GRB progenitors as described by the kinematic model of Stone, Loeb, \& Berger (2013).
\end{abstract}

Keywords: gamma ray: burst - methods: data analysis - accretion

\section{INTRODUCTION}

Several lines of evidence suggest that short duration gamma-ray bursts (hereafter, SGRBs; durations $T_{90} \lesssim$ 2-3 s), or at least a sizable fraction of them, have a cosmological origin and are the electromagnetic counterpart to the coalescence of compact binary systems, such as double neutron stars (NS) or neutron star and black hole (BH; e.g., see Nakar 2007; Berger|2011 for reviews; see also Fong \& Berger 2013; Berger et al. 2013; Tanvir et al. 2013). During the merging, an accretion disk is thought to be produced by the tidal disruption of a NS around a more compact NS or before a NS is swallowed by a BH. Either way, eventually the system evolves towards the formation of a $\mathrm{BH}$ with a debris torus around it. The resulting neutrino-cooled accretion flow leads the hyperaccreting $\mathrm{BH}$ to develop a collimated outflow into a pair of anti-parallel jets (e.g., see Lee \& Ramirez-Ruiz 2007).

A potential means to distinguish between NS-NS and NS-BH mergers concerns the signature of the disk and jet precession in the electromagnetic signal, i.e. the SGRB itself. In the case of a NS-BH merger, precession is expected for a tilted disk and jet due to Lense-Thirring torques from the BH spin (Stone, Loeb, \& Berger 2013 and references therein). These authors (hereafter, SLB13) assumed thick disks precessing as solid body rotators and built upon numerical relativity simulations of this kind of mixed mergers. According to their results, for a reasonable set of values in the parameter space, i.e. $\mathrm{BH}$ spin and mass, disk viscosity, misalignment angle between the accretion disk and the $\mathrm{BH}$ equatorial plane, a quasiperiodic modulation in the $\gamma$-ray signal is to be expected for a sizable fraction of NS-BH mergers. The predicted precession period $T_{\mathrm{p}}$ increases with time proportionally to $t^{4 / 3}$ due to viscous spreading of the disk

\footnotetext{
${ }^{1}$ Department of Physics and Earth Sciences, University of Ferrara, via Saragat 1, I-44122, Ferrara, Italy

2 INAF-IASF Bologna, via Gobetti 101, I-40129, Bologna, Italy
}

and, for a given mixed compact system, starts from a few tens ms at the beginning of the SGRB, and ends with about one order of magnitude longer values. The average expected number of cycles is just a few, typically $N_{\text {cycles }} \lesssim 10$. In all scenarios they considered, these two observables lie in the range $4.5 \lesssim\left\langle N_{\text {cycles }}\right\rangle \lesssim 7.5$ and $30 \mathrm{~ms} \lesssim\left\langle T_{\mathrm{p}}\left(t_{1 / 2}\right)\right\rangle \lesssim 100 \mathrm{~ms}$, where $T_{\mathrm{p}}\left(t_{1 / 2}\right)$ is the half-way precession period for a given merger.

The aim of this letter is to search for this kind of quasiperiodic signal in the data of the brightest SGRBs detected with the Fermi Gamma-ray Burst Monitor (GBM; Meegan et al. 2009), the Swift Burst Alert Telescope (BAT; Barthelmy et al. 2005a), and the Compton Gamma Ray Observatory Burst And Transient Source Experiment (BATSE; Paciesas et al. 1999), exploiting the exquisite time resolution available with these instruments. This search offers the only direct way to observationally distinguish between the two classes of progenitors based on their electromagnetic emission and naturally complements the forthcoming gravitational wave studies. The paper is organized as follows: data selection is described in Section 2. The technique we set up to carry out a dedicated search is outlined in Section 3 . Results and their discussion follow in Sections 4 and 5 . respectively.

\section{DATA SELECTION}

\subsection{Sample selection}

We took all the events observed by the Fermi/GBM from July 2008 to December 2012. For each GRB we extracted and summed the 1-ms light curves of the two most illuminated NaI detectors in the $8-1000 \mathrm{keV}$ energy band with the HEASOFT package (v6.12) following the Fermi team threads. ${ }^{3}$ Light curves affected by spikes due to the interactions of high-energy particles with the spacecraft were rejected (Meegan et al. 2009). We derived the $T_{90}$ and $T_{5 \sigma}$ time intervals, where the bound-

\footnotetext{
${ }^{3}$ http://fermi.gsfc.nasa.gov/ssc/data/analysis/scitools/gbm_grb_analysis.htm
} 
aries of the latter correspond to the first and the last bin whose counts exceed the $5 \sigma$ signal threshold above background.

We selected the SGRBs by requiring $T_{90}<3 \mathrm{~s}^{4}$, and ended up with 160 GRBs, 18 out of which having a minimum signal-to-noise $(\mathrm{S} / \mathrm{N})$ ratio of 20 , as computed over the $T_{5 \sigma}$ interval. As far as the $T_{90}$ distribution is concerned, our selected sample of $\mathrm{S} / \mathrm{N}>20$ SGRBs is representative of the full sample of SGRBs, as suggested by a Kolmogorov-Smirnov test.

The same selection criteria were applied to the Swift/BAT sample using all the events detected up to early June 2013 . We found 30 GRBs with $T_{90}<3 \mathrm{~s}$, 12 out of which passed the final $\mathrm{S} / \mathrm{N}>20$ threshold. The mask-weighted light curves had previously been extracted from the event files following the BAT team threads and concern the 15-150 keV detector passband. In addition to the $1-\mathrm{ms}$ light curves, for the two brightest events of the sample, namely 051221A and 120323A, we used $0.1 \mathrm{~ms}$ resolution, to explore the very highfrequency behavior.

From an initial sample of 61 BATSE SGRBs with high $\mathrm{S} / \mathrm{N}$ we excluded all the cases for which the time-tagged event (TTE) data did not cover the entire profile. Unfortunately, several bright bursts were excluded, because the onboard memory could record only up to 32,768 events around the trigger time. Consequently, we were left with 14 SGRBs whose profiles were extracted in the 20-2000 keV energy range.

Summing up, our final sample includes 44 (18 Fermi, 12 Swift, and 14 CGRO) SGRBs with high S/N (>20). A finer subdivision of the final sample is provided in the following section, aimed at establishing how genuinely short each selected burst is.

\subsection{Short vs. intermediate GRBs}

Evidence for the existence of a third group of GRBs with intermediate durations and hardness ratios between short and long ones was found by several authors for different data sets (e.g., Horváth 1998; Mukherjee et al. 1998; Horváth et al. 2008; Huia et al. 2009; Rípa et al. 2009; Horváth 2009; but see also Koen \& Bere 2012). In this context, we adopted the classification procedures obtained by Horváth et al. (2006) for CGRO/BATSE and by Horváth et al. (2010) for Swift/BAT to assess the nature of our selected sample of bursts, based on the combination of hardness ratio (HR) and $T_{90}$. We assigned each GRB a probability $p$ of belonging to the short group through the "indicator function", out of the three classes: short, intermediate, and long. As expected, all GRBs had negligible probability of belonging to the long group. We defined as "truly SGRB" (T-SGRB) the GRBs with $p>0.9$. The GRBs with $0.8<p<0.9$ are defined as "likely SGRB" (L-SGRB), whereas the remaining cases $(p<0.8)$ were conservatively classified as "possibly intermediate" (P-IGRB). Actually, several members of the P-IGRB group are more likely to be genuine short than intermediate bursts. However, our

\footnotetext{
4 The usual boundary value of $T_{90}=2 \mathrm{~s}$, which was inherited from the BATSE catalog, must not be taken too strictly, the two populations of short and long being partially overlapped. Moreover, this value strongly depends on the detector passband and triggering criteria, as proven by Swift/BAT, which detected several SGRBs with $T_{90}>2 \mathrm{~s}$ (e.g. Barthelmy et al. 2005b).
}

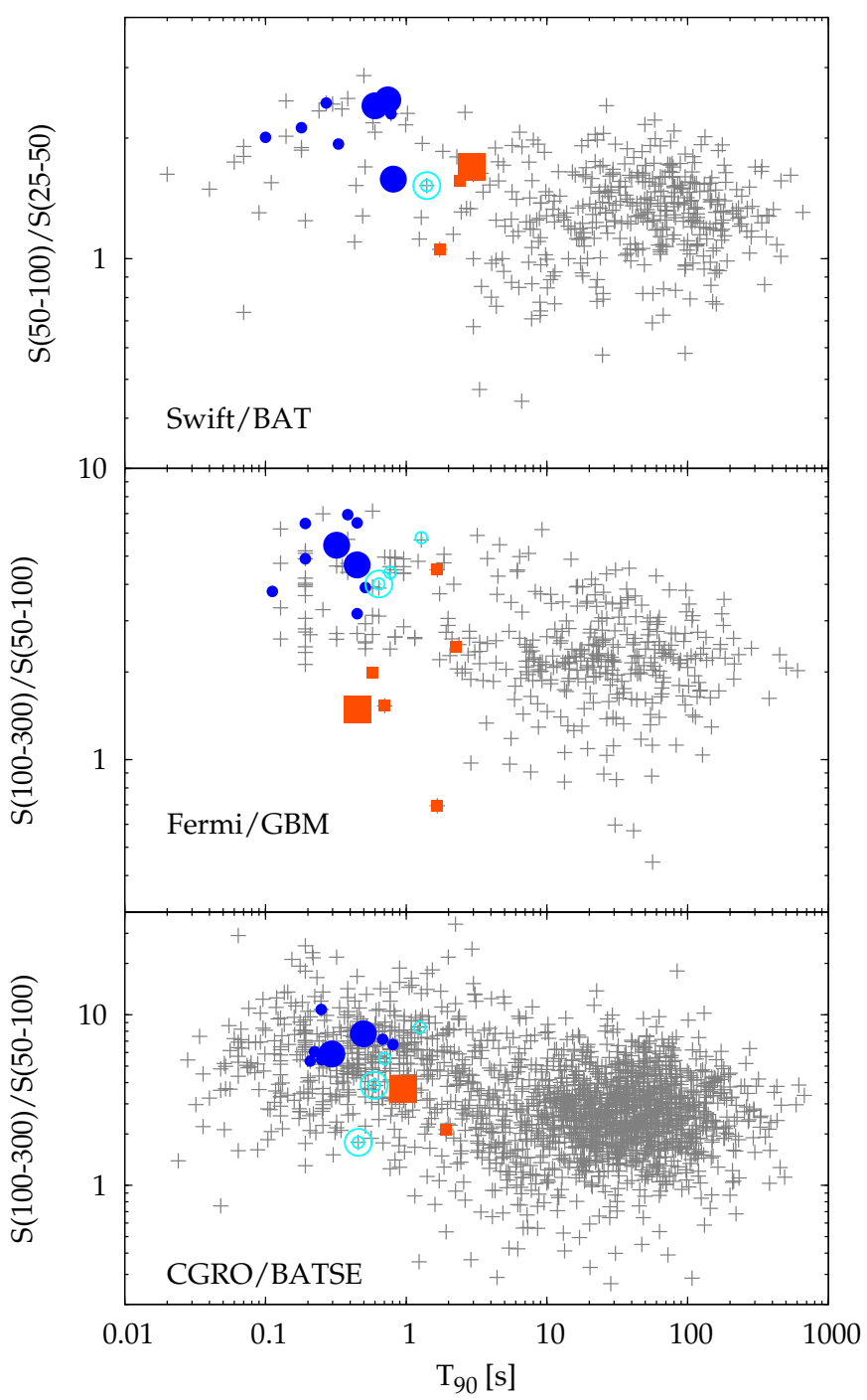

Figure 1. HR- $T_{90}$ diagram for the three data sets: Swift/BAT (top), Fermi/GBM (mid), CGRO/BATSE (bottom). Each panel shows other catalog GRBs (crosses) for comparison. Filled circles, empty circles, and squares correspond to T-SGRBs, L-SGRBs, and P-IGRBs, respectively. Big (small) symbol sizes refer to whether each GRB can (cannot) provide useful constraints on the possible presence of pulsations using the stretched PDS technique (Section [3).

choice was aimed at assuring the least possible contamination with ambiguous cases. Figure 1 shows the HR$T_{90}$ diagram for the three different data sets: each panel compares the properties of our selected GRBs with those of the corresponding catalog: Sakamoto et al. (2011) for Swift/BAT, Paciesas et al. (2012) and Goldstein et al. (2012) for Fermi/GBM, and Paciesas et al. (1999) for CGRO/BATSE. The HR values for the Swift/BAT sample were calculated as the fluence ratio in the bands $(50-100 \mathrm{keV}) /(25-50 \mathrm{keV})$ as in Sakamoto et al. (2011), while $(300-100 \mathrm{keV}) /(50-100 \mathrm{keV})$ was adopted for the Fermi/GBM, and CGRO/BATSE sets. To compute the membership probability for the GRBs detected with the Fermi/GBM, we used the same parameters used for $C G R O /$ BATSE owing to the similar energy passbands. Although in principle this may lead to some misclassified Fermi/GBM GRBs, in practice the two Fermi T-SGRBs 
appear to be robustly so (big filled circles in the mid panel of Fig. 11).

\section{DATA ANALYSIS PROCEDURE}

We studied the power density spectrum (PDS) of each light curve in two different ways. PDS were calculated adopting the Leahy normalization (Leahy et al. 1983). To fit the PDS we used the technique set up by Vaughan (2010) based on a Bayesian treatment with Markov Chain Monte-Carlo techniques. Two analytical models were assumed to describe the PDS continuum: a simple power-law plus constant (hereafter, PL),

$$
S_{\mathrm{PL}}(f)=N f^{-\alpha}+B
$$

or a broken power-law plus constant (hereafter, BPL),

$$
S_{\mathrm{BPL}}(f)=N\left[1+\left(\frac{f}{f_{\mathrm{b}}}\right)^{\alpha}\right]^{-1}+B \quad,
$$

whose low-frequency index is fixed to zero. In either model the constant term accounts for the uncorrelated statistical (white) noise. A likelihood ratio test is used to establish the best model for each PDS. This technique is particularly suitable to the temporal signal of SGRBs, because it searches for (quasi)periodic features superposed to a red-noise process and, as such, can confidently estimate both the best fit parameters of the PDS continuum and the significance of possible features superposed to it, taking into account the uncertainties of the model in a self-coherent way. Moreover, the thresholds for possible periodic features correspond to 2 and $3 \sigma$ (Gaussian) probabilities of a statistical fluctuation and already account for the multi-trial search over the whole range of explored frequencies in each individual PDS. It is worth noting that power approximately fluctuates around the model according to a $\chi^{2}$ distribution with 2 degrees of freedom, i.e. more wildly than a Gaussian variate. Actually, the true distribution deviates from a pure $\chi^{2}$ in that the model itself is affected by uncertainties. This is properly taken into account by the procedure in determining the threshold for a given significance (see Vaughan 2010 and references therein for further details).

The first search was performed on the observed light curves with uniform binning of $1 \mathrm{~ms}$ as they were observed. Hereafter, times are referred to the detector trigger time. We carried out the same analysis in two different time intervals: i) from -3 to $+3 \mathrm{~s}$; ii) over the $T_{5 \sigma}$ interval. For the BATSE sample the analysis was carried out just over the $T_{5 \sigma}$ intervals due to the limited memory of TTE data. The two choices correspond to a fixed temporal range (and, therefore, equal frequency resolution) and to a $\mathrm{S} / \mathrm{N}$-driven scheme, respectively. For three GRBs, namely 110705A, 120323A, and 130603B, we chose a time interval of $2 \mathrm{~s}$, spanning from -1 to $+1 \mathrm{~s}$ instead of the $T_{5 \sigma}$, to properly model the continuum shape. For $051221 \mathrm{~A}$ and $120323 \mathrm{~A}$ we manually selected the time intervals where the analysis was carried out to exploit at the full the $0.1-\mathrm{ms}$ time resolution available in these cases: from -0.80 to $+1.20 \mathrm{~s}$, and from -0.01 to $+0.87 \mathrm{~s}$, respectively. These intervals were chosen to optimize the search for possible signals. Hereafter, we refer to this search as the canonical one, since it does not modify the light curves so as to account for the increasing precession period expected by SLB13. Figure[2] shows

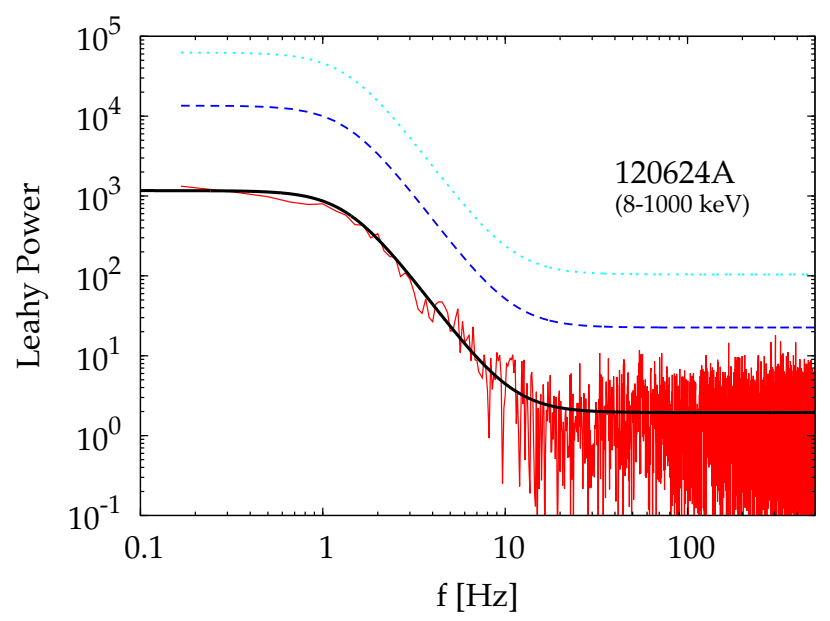

Figure 2. The PDS of GRB $120624 \mathrm{~A}$ fitted using a Bayesian approach. The black solid line represents the best fit model (BPL in this case) while dashed and dotted lines give the probability thresholds at $4.5 \%$ and $0.27 \%$ to find a statistical fluctuation higher than these levels over the whole PDS, respectively. Confidence levels account for the multi-trial frequencies searched within a given PDS.

an example of PDS with the best fit model. Analogous searches which were already performed in the $\mathrm{kHz}$ frequency range in previous data sets of SGRBs, provided only upper limits to the amplitude of possible pulsations (Kruger, Loredo, \& Wasserman 2002). In the absence of any positive detection of periodic signal, we derived the $2 \sigma$ upper limits to the amplitude of detectable periodic pulsations for the frequency range of 10-30 Hz. We expressed this value in terms of fractional amplitude by normalizing the amplitude limit to the peak count rate of each GRB.

We also performed a second, more sensitive search on the PDS of the same light curves after a proper stretching of the time axis. To this aim, we devised a technique which was tailored for the expected signal. For each GRB, we took the $T_{5 \sigma}$ interval boundaries and associated two corresponding precession periods: let $t_{0}$ and $t_{1}$ the start and end times of the $T_{5 \sigma}$ interval and let $T_{p, 0}$ and $T_{p, 1}$ the corresponding precession periods, respectively. We stretched the time axis according to the continuously increasing $T_{p}$ as described by Eq. (3)

$$
T_{p}(t)=T_{p, 0}\left(1+\frac{t-t_{0}}{t_{\mathrm{s}}}\right)^{4 / 3},
$$

where the constant $t_{\mathrm{s}}$ is defined as

$$
t_{\mathrm{s}}=\frac{t_{1}-t_{0}}{\left(T_{p, 1} / T_{p, 0}\right)^{3 / 4}-1} .
$$

The values of $T_{p, 0}$ and $T_{p, 1}$ were chosen so as to match the typical values obtained by SLB13 (typically values were $T_{p, 0}=0.01$ and $T_{p, 1}=0.6 \mathrm{~s}$ ).

We calculated the new count rates in each of the new temporal bins starting from the original photon arrival times at the detector. Earlier bins at $t<t_{0}$ were left unaffected. We attributed a fictitious duration of $1 \mathrm{~ms}$ to the new bins. We made sure the new bins corresponded to a number of 5 bins per precession period. This automatically implies that a possible quasiperiodic pulsation such as that described by Eq. (3) should correspond to a frequency $5 / 2=2.5$ times as small as the Nyquist one (i.e., $200 \mathrm{~Hz}$ in our case) in the stretched PDS. 


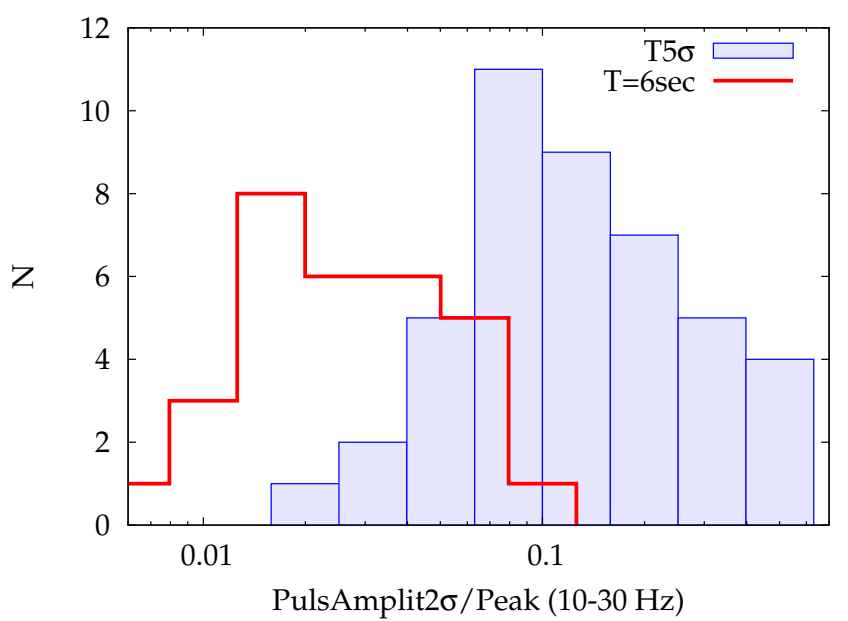

Figure 3. Distribution of the minimum detectable pulsation amplitude normalized to peak in the canonical PDS search. Two cases are shown: fixed time (solid) and $5 \sigma$ (shaded) intervals. They refer to the $10-30 \mathrm{~Hz}$ frequency range.

For each SGRB of our data set, we preliminarily carried out the same analysis on a set of synthetic curves which were derived from a smoothed version of the original light curve of the SGRB. The smoothed version was then modulated with different values of fractional amplitude with a periodic signal with a period varying according to Eq. (3). For each SGRB we determined the minimum amplitude for which the PDS of the synthetic stretched light curve gave a $2-\sigma$ detection. We also searched the synthetic PDS adopting slightly different trial $T_{p, 0}$ and $T_{p, 1}$ from the exact values used to build the corresponding stretched curves. As a result, the detection did not crucially depend on the choice of trial $T_{p, 0}$ and $T_{p, 1}$ within a given range. This check is important since this is the case for real curves for which the possible true periods are unknown a priori. Further details on how synthetic light curves were generated and on the calibration of this technique are given in Appendix A Hereafter, we refer to this search as the stretched PDS one.

\section{RESULTS}

The canonical search identified just a couple of SGRBs with power exceeding the $2 \sigma$ threshold (Gaussian units) in one frequency bin each. The chance probability of a $2 \sigma$ fluctuation occurring within a given PDS is $4.5 \%$. Out of 44 different PDS, the expected number of $>2 \sigma$ fluctuations is 1.98 , i.e. in agreement with the observed number of two cases. Hence, no evidence for the presence of periodic or quasiperiodic signal was found. In the absence of detection, for each GRB we derived a $2 \sigma$ upper limit to the fractional amplitude averaged out over the frequency range of interest, i.e. from 10 to $30 \mathrm{~Hz}$. The amplitude is normalized to the peak count rate of each SGRB. The average minimum detectable amplitude depends on the time interval the PDS is calculated: it clusters around a $3 \%(17 \%)$ of the peak for the fixed $(5 \sigma)$ time interval (Fig. 3).

Likewise, we did not find any evidence for the quasiperiodic signals in the stretched PDS search. However, as the calibration on synthetic curves has shown, we could obtain useful upper limits to the pulsational amplitude for the four, five, and five SGRBs with highest S/N detected by Fermi, Swift, and CGRO, respectively. This

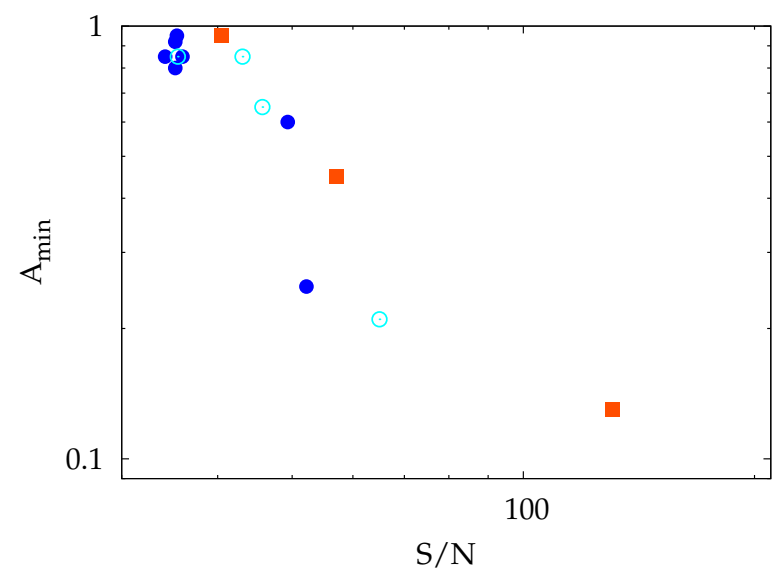

Figure 4. Minimum detectable fractional amplitude for an increasing precession period for 14 SGRBs, as determined from simulations in the stretched PDS search. Same symbols as in Figure 1 are used.

reduced sensitivity with respect to the canonical search is a consequence of the low number of expected cycles coupled with the statistical quality of the data. Figure 4 displays the $2 \sigma$ upper limits to the fractional amplitude for a modulation with an increasing precession period superposed to the overall profile of each SGRB as in Eq. (3) as a function of $\mathrm{S} / \mathrm{N}$ for these 14 events. With reference to the short/intermediate classification provided in Section 2.2, 7 out of these 14 GRBs are T-SGRBs, while the remaining 4 and 3 are L-SGRBs and P-IGRBs, respectively. As shown in Figure 4, even neglecting the $\mathrm{P}$-IGRB group our results do not change in essence, although the reduced number of events demands caution in generalizing them to larger samples of GRBs. The burst with the highest $\mathrm{S} / \mathrm{N}$ and most stringent upper limit to the fractional amplitude corresponds to GRB 120323A detected with Fermi/GBM and it is a P-IGRB, so the probability of being a misclassified intermediate GRB is not negligible. Still, it is worth noting that its probability of being a genuine SGRB is $78 \%$ against a mere $22 \%$ of being intermediate.

Although the QPO search has given negative results, an interesting product of the canonical search is the continuum properties for an ensemble of bright SGRBs, which is studied here for the first time. Figure 5 shows the distribution of the power-law indices for both PL and the BPL models, upon selection of the most accurately measured values $(|\sigma(\alpha)|<0.5)$. A comparison with analogous results obtained on a sample of long Fermi/GBM GRBs (Dichiara et al. in prep.) shows no outstanding difference in the power-law index distribution between short and long GRBs. Yet the small number of SGRBs lacks in sensitivity to reveal fine differences.

For the SGRBs whose PDS is best fit with a broken power-law, the break frequency is mostly connected to the overall duration of the main spike, whose timescale is predominant in the total PDS of SGRBs.

\section{DISCUSSION AND CONCLUSIONS}

The canonical search for periodic or quasiperiodic signal did not yield any detection, in agreement with previous analogous searches (Kruger, Loredo, \& Wasserman 2002), down to a limiting peak-normalized amplitude which is typically around 10-20\% when the PDS is cal- 


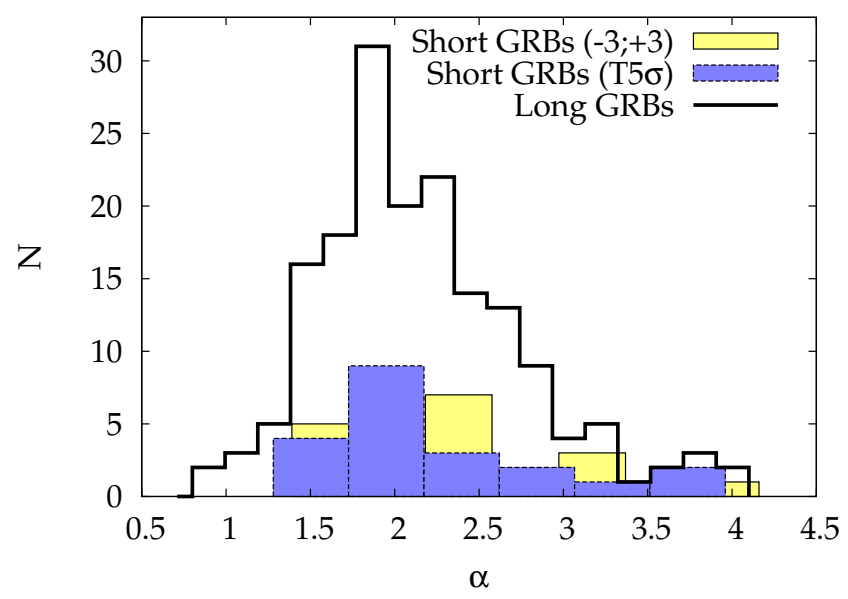

Figure 5. Distribution of the PDS slope as derived from the $[-3 ; 3] \mathrm{s}$ interval (light shaded), and the $5 \sigma$ interval (dark shaded). Also shown is the same distribution for a sample of 170 long GRBs (solid line; Dichiara et al., in prep.).

culated over the $5 \sigma$ time interval.

In addition, we devised and calibrated a technique to detect the signature of a periodic signal potentially hidden within the time profiles of some SGRBs, characterized by a continuously increasing period, from a few tens ms up to a fraction of a second or so throughout the duration of SGRB. This kind of signal has theoretically been predicted in the case of a mixed merger (NS-BH), where the tilted jet and accretion disk with respect to the $\mathrm{BH}$ spin is expected to cause the jet precession and a periodic gamma-ray signal in the prompt emission such as that described above (SLB13). Likewise, no significant detection at $2 \sigma$ out of a sample of 44 SGRBs was obtained by our tailored technique, named the stretched PDS search, either. However, we could extract useful upper limits to the fractional amplitude of such a modulated signal for $14 \mathrm{GRBs}$, with values distributed from 10 to $90 \%$. When we exclude the 3 GRBs which appear to have a non-negligible $(p>0.2)$ probability of belonging to the intermediate duration group, the results do not change in essence. The reduced sensitivity of the stretched PDS search compared with that of the canonical one is due to smaller numbers of expected cycles, which couple with a more critical dependence on $\mathrm{S} / \mathrm{N}$, as revealed by the synthetic curves used for calibration.

An interesting outcome of our canonical PDS search concerns the continuum properties of the PDS for an ensemble of bright SGRBs (see Table in electronic format).
Unlike the case for long GRBs (e.g., see Dichiara et al. 2013 and references therein), this is the first time we could usefully study these properties for SGRBs, whose study has been hampered so far by lower S/N with respect to long GRBs. This was also made possible by the Bayesian procedure that was recently proposed by Vaughan (2010) to properly model the PDS of time series affected by a strong red noise component, such as the case of SGRBs' time histories (e.g., see Huppenkothen et al. 2013). Two alternative models were adopted: a simple or a broken power-law in addition to the white noise constant. A preliminary comparison with the analogous properties of a sample of bright long GRBs (Dichiara et al. in prep.) reveals no striking difference between the two power-law index distributions (Fig. 5). Regardless of the PDS continuum interpretation, this may suggest a common general mechanism which rules the shock formation and the gamma-ray emission production.

The implications of our results do not allow us to rule out the physical scenario envisaged by SLB13 as the possible interpretation of the prompt emission of SGRBs for two main reasons. First of all, the sample of SGRBs for which our non-detection is meaningful is still statistically too small to draw firm conclusions. This is even more so when one neglects the few GRBs which could belong to the intermediate duration group. Secondly, the possibility that the few cases of interest could correspond to either other kind of mergers, such as NS-NS, or mixed mergers with unfavorable space parameters, such as the accretion disk viscosity or the misalignment angle between jet axis and $\mathrm{BH}$ spin, is not negligible for just a few cases. Furthermore, according to the recent physical classification proposed by Bromberg et al. (2013), there could be collapsar events disguised as SGRBs, whose presence could partially explain the observed lack of evidence for the pulsations expected for NS-BH mergers. Nonetheless, in addition to being the first attempt of a dedicated search on a valuable data set, our analysis indicates that such mixed systems might not be a dominant fraction among the population of currently detected SGRBs, at least as envisaged in the model by SLB13. A definitive answer will come from a larger sample with comparable statistical quality in combination with the wealth of information that will be independently gathered through the study of gravitation wave radiation.

\section{APPENDIX}

\section{CALIBRATION OF THE STRETCHED PDS SEARCH}

For each SGRBs we carried out a series of simulations aimed at calibrating the sensitivity of our stretched PDS search. We first binned the original curve to a rough resolution so as to reduce the high-frequency variability (both real and statistical fluctuations). The smoothed version of the light curve was then obtained by interpolation of the coarse binned curve by means of $\mathrm{C}$-splines. To simulate the predicted periodicity we modulated a smoothed version of the original light curve with a sinusoidal signal assuming the temporal evolution of $T_{p}$ of Eq. (3). Specifically, to obtain the synthetic light curves we preliminarily had to calculate the pulsational phase as a function of time, $\phi(t)$. Since $T_{p}$ continuously varies with time, we had to integrate the infinitesimal relation $d \phi=2 \pi d N=2 \pi d t / T_{p}$, where $d N$ is the infinitesimal increment to the total number of cycles starting from $t_{0}$. Using Equation (3) one obtains

$$
\phi(t)=2 \pi \int_{t_{0}}^{t} \frac{d t^{\prime}}{T_{p}\left(t^{\prime}\right)}=\frac{6 \pi t_{\mathrm{s}}}{T_{p, 0}}\left[1-\left(1+\frac{t-t_{0}}{t_{\mathrm{s}}}\right)^{-1 / 3}\right] .
$$


Equivalently, the number of cycles at time $t, N(t)$ is given by

$$
N(t)=\frac{\phi(t)}{2 \pi}=\frac{3 t_{\mathrm{s}}}{T_{p, 0}}\left[1-\left(1+\frac{t-t_{0}}{t_{\mathrm{s}}}\right)^{-1 / 3}\right] .
$$

The final number of cycles is given by Eq. (A2) at $t=t_{1}$ and can be conveniently expressed as

$$
N=\frac{\left(t_{1}-t_{0}\right)}{T_{p, 0}} \frac{3 x^{3}}{1+x+x^{2}},
$$

where we defined $x=\left(T_{p, 0} / T_{p, 1}\right)^{1 / 4}$. The trivial case of constant periodicity $\left(T_{p, 1}=T_{p, 0}\right)$ is easily recovered, being $N=\left(t_{1}-t_{0}\right) / T_{p, 0}$. Finally, statistical noise was added to the synthetic light curves, which were then processed exactly in the same as real curves according to the stretched PDS search described in Section 3.

This work was supported by PRIN MIUR project on "Gamma Ray Bursts: from progenitors to physics of the prompt emission process", P. I. F. Frontera (Prot. 2009 ERC3HT). The useful comments of the anonymous referee are gratefully acknowledged. We thank Raffaella Margutti for reading the manuscript and for useful comments.

\section{REFERENCES}

Barthelmy, S. D., et al. 2005a, Space Sci. Rev., 120, 143

Barthelmy, S. D., et al. 2005b, Nature, 438, 994

Berger, E., 2011, New A Rev., 55, 1

Berger, E., W. Fong, \& R. Chornock, 2013, ApJ, 744, L23

Bromberg, O., Nakar, E., Piran, T., Sari, R., 2013, ApJ, 764, 179

Dichiara, S., Guidorzi, C., Amati, L., Frontera, F., 2013, MNRAS, 431, 3608

Fong, W., Berger, E., 2013, submitted (arXiv 1307.0819)

Goldstein, A., et al., 2012, ApJS, 199, 19

Horváth, I., 1998, ApJ, 508, 757

Horváth, I., Balázs, L. G., Bagoly, Z., Ryde, F., Mészáros, A., 2006, A\&A, 447, 23

Horváth, I., Balázs, L. G., Bagoly, Z., Veres, P., 2008, A\&A, 489, L1

Horváth, I., 2009, Ap\&SS, 323, 83

Horváth, I., et al., 2010, ApJ, 713, 552

Huja, D., Mészáros, A., Rípa, J., 2009, A\&A, 504, 67

Huppenkothen, D., et al., 2013, ApJ, 768, 87
Koen, C., Bere, A., 2012, MNRAS, 420, 405

Kruger, A. T., Loredo, T. J., Wasserman, I., 2002, ApJ, 576, 932

Leahy, D. A., Darbro, W., Elsner, R. F., Weisskopf, M. C.,

Sutherland, P. G., Kahn, S., \& Grindlay, J. E., 1983, ApJ, 266, 160, L83

Lee, W. H., Ramirez-Ruiz, E., 2007, New Journal of Physics, 9, 17

Meegan, C. et al., 2009, ApJ, 702, 791

Mukherjee, S., et al., 1998, ApJ, 508, 314

Nakar, E., 2007, Phys. Rep., 442, 166

Paciesas, W. S., et al., 1999, ApJS, 122, 465

Paciesas, W. S., et al., 2012, ApJS, 199, 18

Rípa, J., et al., 2009, A\&A, 498, 399

Sakamoto, T., et al., 2011, ApJS, 195, 2

Stone, N., Loeb, A, \& Berger, E, 2013, Phys. Rev., D87, 084053 (SLB13)

Tanvir, N.R., et al., 2013, Nature, 500, 547

Vaughan, S., 2010, MNRAS, 402, 307 
Table 1

Best-fitting model and parameters for each SGRB of the total sample.

\begin{tabular}{|c|c|c|c|c|c|c|c|c|c|c|c|c|c|c|}
\hline$\overline{\text { GRB }}$ & Model & $\overline{l o g} N$ & $\begin{array}{c}\log f_{\mathrm{b}} \\
(\mathrm{Hz})\end{array}$ & $\bar{\alpha}$ & $\bar{B}$ & $\overline{p\left(T_{R}\right)^{\mathrm{a}}}$ & $p_{\mathrm{AD}}{ }^{\mathrm{b}}$ & $\overline{p_{\mathrm{KS}}{ }^{\mathrm{c}}}$ & PulseA $A_{2 \sigma} /$ Peak & $T_{90}$ & $t^{\text {start }}$ & $t^{\text {stop }}$ & $\overline{\mathrm{HR}^{\mathrm{g}}}$ & $\overline{p(\text { Short })}$ \\
\hline $051221 A^{\mathrm{d}}$ & $\mathrm{BPL}$ & $4.487_{-1.278}^{+7.780}$ & $-0.732^{+0.838}$ & $1.717_{-010}^{+0.119}$ & $1.812_{-0.076}^{+0.072}$ & 0.138 & 0.654 & 0.884 & 0.014 & 1.370 & -3.000 & +3.000 & $1.522 \pm 0.074$ & 0.841 \\
\hline $060313^{\mathrm{d}}$ & $\mathrm{BPL}$ & $3.066_{-0.350}^{+0.518}$ & $0.089_{-0.375}^{+0.276}$ & $1.968_{-0.221}^{+0.110}$ & $1.925_{-0.074}^{+0.076}$ & 0.428 & 0.385 & 0.366 & 0.019 & 0.818 & -3.000 & +3.000 & $2.491 \pm 0.151$ & 0.999 \\
\hline $061201^{d}$ & PL & $1.569_{-0.179}^{+0.193}$ & - & $1.398_{-0.222}^{+0.239}$ & $2.025_{-0.070}^{+0.074}$ & 0.942 & 0.997 & 0.999 & 0.042 & 0.827 & -3.000 & +3.000 & $2.299 \pm 0.299$ & 0.999 \\
\hline $080426^{\mathrm{d}}$ & PL & $1.555_{-0.196}^{+0.179}$ & - & $2.507_{-0.512}^{+0.522}$ & $1.954_{-0.060}^{+0.070}$ & 0.535 & 0.845 & 0.775 & 0.043 & 2.019 & -3.000 & +3.000 & $1.055 \pm 0.12$ & 0.123 \\
\hline $081107^{\mathrm{e}}$ & $\mathrm{PL}$ & $1.209_{-0.216}^{+0.1926}$ & - & $2.274_{-0.455}^{+0.585}$ & $1.958_{-0.059}^{+0.060}$ & 0.233 & 0.877 & 0.795 & 0.065 & 1.792 & -3.000 & +3.000 & $0.695 \pm 0.200$ & 0.006 \\
\hline $081209^{\mathrm{e}}$ & $\mathrm{BPL}$ & $1.902_{-0.271}^{+0.316}$ & $0.371_{-0.323}^{+0.246}$ & $2.478_{-0.632}^{+0.829}$ & $1.985_{-0.063}^{+0.069}$ & 0.625 & 0.488 & 0.664 & 0.019 & 0.960 & -3.000 & +3.000 & $4.897 \pm 0.657$ & 0.989 \\
\hline $081216^{\mathrm{e}}$ & $\mathrm{BPL}$ & $2.342_{-0.225}^{+0.271}$ & $0.385_{-0.160}^{+0.131}$ & $6.775_{-2.928}^{+5.190}$ & $1.981_{-0.057}^{+0.059}$ & 0.819 & 0.888 & 0.807 & 0.019 & 1.152 & -3.000 & +3.000 & $4.390 \pm 0.271$ & 0.854 \\
\hline $081223^{\mathrm{e}}$ & PL & $1.622_{-0.198}^{+0.216}$ & - & $2.368_{-0.454}^{+0.938}$ & $1.980_{-0.059}^{+0.065}$ & 0.148 & 0.927 & 0.627 & 0.042 & 1.536 & -3.000 & +3.000 & $1.988 \pm 0.953$ & 0.747 \\
\hline $090108^{\mathrm{e}}$ & $\mathrm{BPL}$ & $2.510_{-0.300}^{+0.1983}$ & $0.130_{-0.205}^{+0.158}$ & $4.692_{-1.432}^{+0.454}$ & $1.982_{-0.061}^{+0.059}$ & 0.797 & 0.499 & 0.559 & 0.024 & 0.768 & -3.000 & +3.000 & $1.531 \pm 0.595$ & 0.500 \\
\hline
\end{tabular}

Note. - Uncertainties on best-fit parameters are given at $90 \%$ confidence. This table is available in its entirety in a machine-readable form in the online journal

${ }^{a} p\left(T_{R}\right)$ is the significance associated to statistic $T_{R}$.

${ }^{\mathrm{b}} p_{\mathrm{AD}}$ is the significance of the Anderson-Darling test.

${ }^{c} p_{\mathrm{KS}}$ is the significance of the Kolmogorov-Smirnov test.

Detected by Swift/BAT

e Detected by Fermi/GBM

f Detected by $C G R O /$ BATSE

g Uncertainty on hardness ratio are given at 1 sigma confidence

${ }^{\mathrm{h}}$ In this case the time interval of PDS extraction is larger then the $T_{5 \sigma}$ interval to fit properly the continuum shape 